\title{
Optimal fuel consumption finite-thrust orbital hopping of aeroassisted spacecraft
}

\author{
Runqi Chai ${ }^{\mathrm{a}}$, Al Savvaris ${ }^{\mathrm{a}}$, Antonios Tsourdos ${ }^{\mathrm{a}}$, Senchun Chai ${ }^{\mathrm{b}, *}$, Yuanqing \\ $\mathrm{Xia}^{\mathrm{b}}$ \\ ${ }^{a}$ School of Aerospace, Transport and Manufacturing, Cranfield University, Bedfordshire, \\ MK43 OAL, United Kingdom \\ ${ }^{b}$ School of Automation, Beijing Institute of Technology, Beijing 100081, PR China
}

\begin{abstract}
In the paper, the problem of minimum-fuel aeroassisted spacecraft regional reconnaissance (orbital hopping) is considered. A new nonlinear constrained optimal control formulation is designed and constructed so as to describe this mission scenario. This formulation contains multiple exo-atmospheric and atmospheric flight phases and correspondingly, two sets of flight dynamics. The constructed continuous-time optimal control system is then discretized via a multi-phase global collocation technique. The resulting discrete-time system is optimized using a newly proposed gradient-based optimization algorithm. Several comparative simulations are carried out and the obtained optimal results indicate that it is effective and feasible to use the proposed multi-phase optimal control design for achieving the aeroassisted vehicle orbital hopping mission.
\end{abstract}

Keywords: Aeroassisted spacecraft, orbital hopping, optimal control, trajectory optimization.

\section{Introduction}

In the past few decades, aeroassisted orbital transfer vehicles have received considerable attention due to their extensive applications in space exploration [1] 3]. One important advantage of using this type of flight vehicle is that it has

\footnotetext{
* Corresponding author

Email addresses: r.chai@cranfield.ac.uk (Runqi Chai), a.savvaris@cranfield.ac.uk (Al Savvaris), a.tsourdos@cranfield.ac.uk (Antonios Tsourdos), chaisc97@bit.edu.cn (Senchun Chai), xia_yuanqing@bit.edu.cn (Yuanqing Xia)
} 
5 the capability to apply the aerodynamic forces and its engine model effectively 1, 4. Early works on developing the aeroassisted spacecraft mainly focus on the propulsion and online guidance systems [5] 7]. For example, in [8], the authors proposed an online minimum energy-loss guidance strategy for the aeroassisted vehicle. Naidu et al. 9] designed a neighbouring optimal guidance scheme 10 for the nonlinear aeroassisted vehicle dynamics. Meanwhile, many important research works focusing on the aeroassisted vehicle orbital transfer have been extensively investigated [4, 10. Specifically, Darby and Rao [11] considered a small-scale spacecraft orbital transfer problem using impulsive thrust. In their work, the entire mission was completed during the space flight. Begum et al.

15 12 designed the aeroassisted orbital transfer trajectory based on the optimal control theory. Different with the work carried out in [11, both the space flight and atmospheric pass were used to complete the mission in [12.

Although the aforementioned research works show the potential feasibility and benefits of using the aeroassisted vehicle for the orbital transfer, less atten-

20 tion has been paid to apply the aeroassisted vehicle for an orbital hopping or regional reconnaissance mission profile. Therefore, in this paper, a new aeroassisted spacecraft orbital hopping problem formulation is proposed and studied. The main objective of this work is to generate the minimum-fuel trajectory for the orbital hopping mission. Then based on the obtained optimal solutions, a 25 better understanding in terms of the performance requirements and the structure of the problem can be gained.

The overall optimal fuel consumption aeroassisted vehicle orbital hopping problem is formulated as a multiple-phase nonlinear optimal control problem. This type of problem is becoming an active topic since the obtained optimal refso erence trajectory can be implemented in various industrial applications [13 16]. To calculate the optimal solution, a typical direct transcription algorithm (e.g. Gauss pseudospectral method [17, 18]) is applied to discretize the vehicle dynamics. In recent years, global collocation techniques have attracted extensive attentions and a large amount of work is being carried out in this field [3]. For 35 example, Fahroo and Ross [19] developed a Chebyshev pseudospectral approach for solving the general Bolza trajectory optimization problems with control and state constraints. In their follow-up work [20, a pseudospectral knotting algorithm was designed so as to solve nonsmooth optimal control problems. The main advantage with pseudospectral methods is that a high approximation ac- 
curacy can be achieved with much less temporal nodes [18, 21]. After generating the optimal solutions, the results are analyzed to show the key features of the constructed problem in the simulation section.

The rest of this paper is organized as follow: In Section 2, a new minimumfuel aeroassisted spacecraft orbital hopping mission is proposed and formulated.

45 In order to guide the vehicle overflying different ground target positions, a series of event sequences are constructed and embedded in the problem formulation. Section 3 gives a brief description in terms of the direct algorithm used to calculate the optimal solution. The main results are provided in Section 4, where comparative simulations verify the effectiveness and feasibility of the proposed design philosophy. The concluding remark is given in Section 5 .

\section{Aeroassisted spacecraft reconnaissance optimal control problem}

The mission scenario investigated in this research focuses on the atmospheric skip hopping, targeting the entry into the atmosphere down to different predetermined positions for observation and gathering of information of inaccessible areas. Once these positions are reached, the spacecraft starts the ascent phase, exiting the atmosphere and returning back to Low Earth Orbit (LEO). During the mission, the aeroassisted spacecraft can fly in either the unpowered exoatmospheric flight, powered exo-atmospheric flight, or unpowered atmospheric flight. The overall mission profile is illustrated in Fig.1.

It is worth noting that as shown in Fig.1, the dashed line phases may repeat several times (e.g. $n-1$ times). This is because in this paper, it is expected for the aeroassisted vehicle to have a multiple-hop trajectory in order to overfly different target regions and complete the reconnaissance mission. An example of a single-hop mission can be found in our previous work [22].

65 2.1. Vehicle equations of motion

The dynamics of the aeroassisted vehicle is modeled as a point mass over a spherical rotating Earth. For the exo-atmospheric flight, the effect caused by aerodynamic forces can be ignored and the differential equations of motion are 


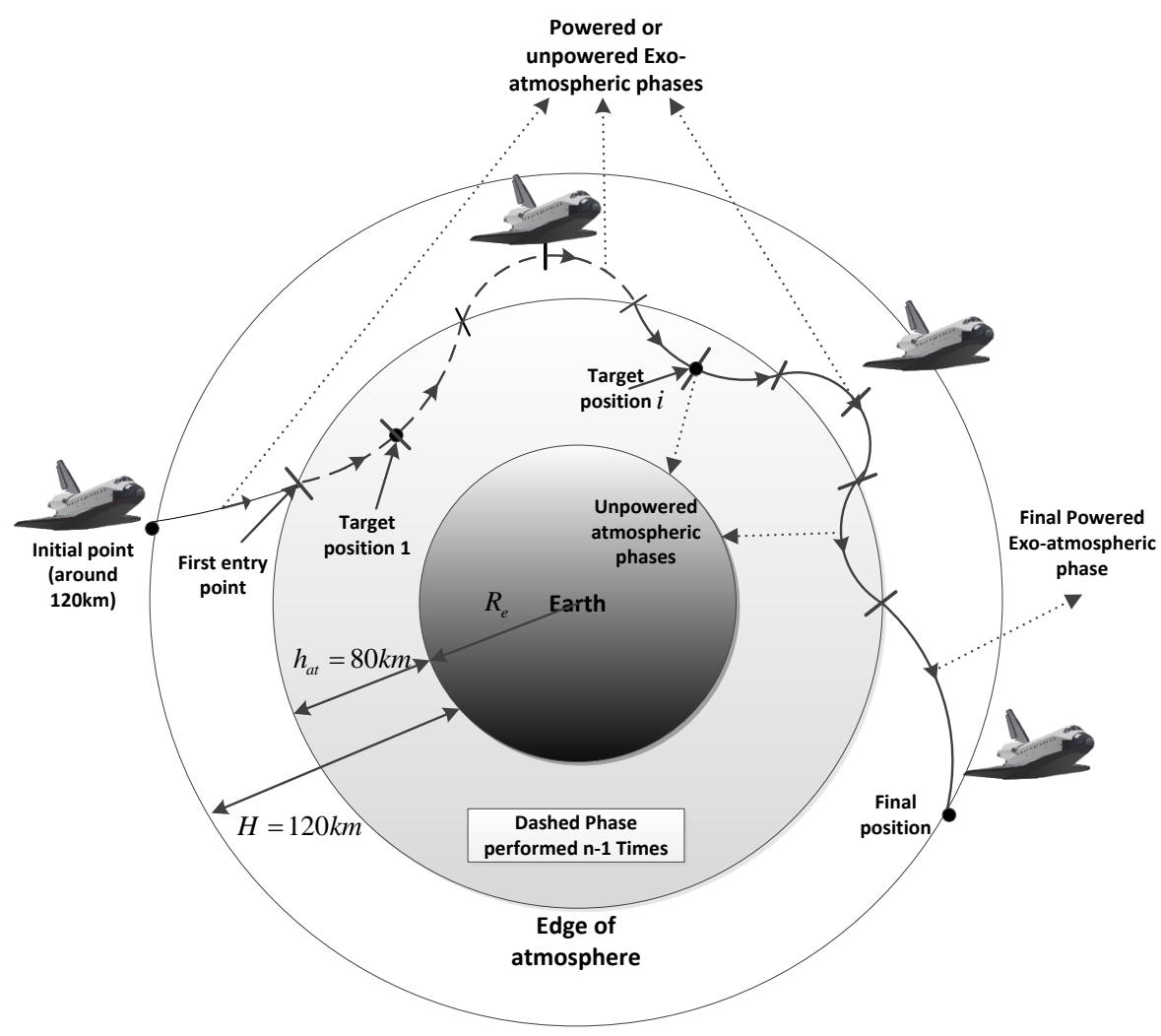

Figure 1: Aeroassisted vehicle orbital hopping mission profile

defined as [23, 24]:

$$
\left\{\begin{array}{l}
\dot{r}=V \sin \gamma \\
\dot{\theta}=\frac{V \cos \gamma \sin \psi}{r \cos \phi} \\
\dot{\phi}=\frac{V \cos \gamma \cos \psi}{r} \\
\dot{V}=\frac{T \cos \alpha}{m}-g \sin \gamma+\omega_{V} \\
\dot{\gamma}=\frac{T \sin \alpha}{m V}+\left(\frac{V^{2}-g r}{r V}\right) \cos \gamma+\omega_{\gamma} \\
\dot{\psi}=\frac{V}{r} \cos \gamma \sin \psi \tan \phi+\omega_{\psi} \\
\dot{m}=-\frac{T}{I_{s p} g}
\end{array}\right.
$$

70

where $r, \theta, \phi, V, \gamma, \psi, m$ represent the radial distance, longitude, latitude velocity, flight-path angle, heading angle and vehicle's mass, respectively. $\alpha$ is the angle of attack and $T$ is the thrust force. During unpowered flight phases, $T$ is set to zero. The gravity $g=\frac{\mu}{r^{2}}$, in which $\mu$ is the gravitational parameter. $I_{s p}$ is the specific impulse. $\omega_{V}, \omega_{\gamma}$ and $\omega_{\psi}$ stand for the contribution of Coriolis 
acceleration and convected acceleration. Their analytical expressions can be given by

$$
\left\{\begin{array}{l}
\omega_{V}=\Omega^{2} r \cos \phi(\sin \gamma \cos \phi-\cos \gamma \sin \psi \cos \psi) \\
\omega_{\gamma}=2 \Omega \cos \phi \sin \psi+\Omega^{2} r \cos \phi(\cos \gamma \cos \phi+\sin \gamma \cos \psi \sin \phi) \\
\omega_{\psi}=\frac{\Omega^{2} r \cos \phi \sin \phi}{\cos \gamma}-2 \Omega(\tan \gamma \cos \psi \cos \phi-\sin \phi)
\end{array}\right.
$$

where $\Omega=7.2921151 e^{-5} \mathrm{rad} / \mathrm{s}$ is the self-rotation rate of the Earth.

During the unpowered atmospheric flight phase, the aerodynamic forces (e.g. aerodynamic lift and drag) should be taken into account. Therefore, the corresponding equations of motion for the aeroassisted spacecraft are formulated as:

$$
\left\{\begin{array}{l}
\dot{r}=V \sin \gamma \\
\dot{\theta}=\frac{V \cos \gamma \sin \psi}{r \cos \phi} \\
\dot{\phi}=\frac{V \cos \gamma \cos \psi}{r} \\
\dot{V}=-\frac{\rho V^{2} S C_{D}}{2 m}-g \sin \gamma+\omega_{V} \\
\dot{\gamma}=\frac{\rho V^{2} S C_{L} \cos \sigma}{2 m V}+\left(\frac{V^{2}-g r}{r V}\right) \cos \gamma+\omega_{\gamma} \\
\dot{\psi}=\frac{\rho V^{2} S C_{L} \sin \sigma}{2 m V \cos \gamma}+\frac{V}{r} \cos \gamma \sin \psi \tan \phi+\omega_{\psi}
\end{array}\right.
$$

where $S$ is the reference area of the vehicle. $\rho=\rho_{0} \exp \frac{r-R_{e}}{h_{s}}$ is the density of the atmosphere, $\rho_{0}$ is the density of the atmosphere at sea-level and $R_{e}$ is the radius of the Earth. $C_{L}=C_{L 0}+C_{L 1} \alpha$ and $C_{D}=C_{D 0}+C_{D 1} \alpha+C_{D 2} \alpha^{2}$ are lift and drag coefficients, where $C_{L 0}, C_{L 1}, C_{D 0}, C_{D 1}$ and $C_{D 2}$ are set as constants.

\subsection{Trajectory event sequence}

For the multiple-phase orbital hopping mission, the trajectory event sequence (multiple hops) can be summarised as follows:

1. The aeroassisted vehicle starts with a powered exo-atmospheric flight phase that starts on the initial position and terminates at a specified altitude $h_{a t}=80 \mathrm{~km}$ where is the assumed edge of the atmosphere;

2. An unpowered atmospheric skip hop phase that starts at the altitude $h_{a t}$, overflights the first ground target position and terminates at the altitude $h_{a t}$;

95 3. An unpowered exo-atmospheric flight that starts at $h_{a t}$ (assumed edge of the atmosphere);

4. A powered exo-atmospheric flight that terminates at the altitude $h_{a t}$. 
The above last three event sequences are repeated $n-1$ times, where $n$ stands for the number of reconnaissance regions and is a mission-dependent the entire mission.

5. An unpowered atmospheric skip entry phase that starts and terminates at the specified altitude point $h_{a t}$.

6. A powered exo-atmospheric flight phase that begins at $h_{a t}$ and flies back to the predesigned terminal conditions.

As suggested in 12, the last two events (5 and 6) are embedded in the multiple-phase orbital hopping event sequences. That is, the spacecraft can have an additional skip entry flight to adjust the attitude and velocity of the vehicle so that the vehicle can have more flexibility to complete the entire mission. Moreover, this will also have positive influences in terms of the solutionfinding process discussed in the simulation section. An overall description of the trajectory event sequence can also be found in Fig.1.

\subsection{Vehicle and mission constraints}

\subsubsection{Boundary conditions}

At the start point of the mission, the initial boundary conditions are given by:

$$
\begin{array}{cccc}
r\left(t_{0}\right)=R_{e}+H & \theta\left(t_{0}\right)=\theta_{0} & \phi\left(t_{0}\right)=\phi_{0} & V\left(t_{0}\right)=V_{0} \\
\gamma\left(t_{0}\right)=\gamma_{0} & \psi\left(t_{0}\right)=\psi_{0} & m\left(t_{0}\right)=m_{0} &
\end{array}
$$

where $t_{0}=0$ is the initial time. $H=120 \mathrm{~km}$ is the initial altitude value. The boundary conditions corresponding to the $i$ th target position are given as follows:

$$
\left\{\begin{aligned}
r\left(t_{i}\right) & =R_{e}+H_{i} \\
\theta\left(t_{i}\right) & =\theta_{i} \\
\phi\left(t_{i}\right) & =\phi_{i} \\
\gamma\left(t_{i}\right) & =\gamma_{i} \\
r\left(t_{f}\right) & =r_{f}
\end{aligned}\right.
$$

120

where $1 \leq i \leq n, t_{i}$ is the time instant when the aeroassisted spacecraft reaches the $i$ th target position. $t_{f}$ stands for the terminal time instant and $r_{f}=R_{e}+H$ is the final altitude value. 


\subsubsection{Box constraints}

During the skip hopping, all the design variables should satisfy the box

125 constraints:

$$
\begin{array}{lll}
r_{\min } \leq r \leq r_{\max } & \theta_{\min } \leq \theta \leq \theta_{\max } & \phi_{\min } \leq \phi \leq \phi_{\max } \\
V_{\min } \leq V \leq V_{\max } & \gamma_{\min } \leq \gamma \leq \gamma_{\max } & \psi_{\min } \leq \psi \leq \psi_{\max } \\
\alpha_{\min } \leq \alpha \leq \alpha_{\max } & \sigma_{\min } \leq \sigma \leq \sigma_{\max } & m_{\min } \leq m \leq m_{\max }
\end{array}
$$

where the subscript min and max represent the lower and upper bounds of the decision variable.

\subsubsection{Interior-point constraints}

Defining $t_{f}^{-}$and $t_{0}^{+}$as the ultimate time instant of a flight phase and the initial time instant of the continuing flight phase, in order to enforce continuity in the design variables at the phase boundaries, the multiple-phase interior-point constraints are then introduced. That is,

$$
\begin{array}{rlrl}
r\left(t_{f}^{-}\right) & =t_{0}^{+} & \theta\left(t_{f}^{-}\right)=\theta_{0}^{+} \\
\phi\left(t_{f}^{-}\right)=\phi_{0}^{+} & V\left(t_{f}^{-}\right)=V_{0}^{+} \\
\gamma\left(t_{f}^{-}\right)=\gamma_{0}^{+} & \psi\left(t_{f}^{-}\right)=\psi_{0}^{+} \\
m\left(t_{f}^{-}\right)=m_{0}^{+} & t_{f}^{-}=t_{f}^{+}
\end{array}
$$

In Eq. (7), since $t$ is considered as a free design variable, an interior-point constraint with respect to the boundary time is introduced (e.g. $t_{f}^{-}=t_{f}^{+}$) to connect the neighbouring two phases.

\subsubsection{Flight path constraints}

The spacecraft reconnaissance mission should satisfy strict path constraints to protect the structure of the vehicle. In both the exo-atmospheric and atmospheric flight phases, the aerodynamic heating $\dot{Q}$ constraint is introduced to the mathematical model and it can be written as [25]:

$$
\dot{Q}=K_{Q} \rho^{0.5} V^{3.07}\left(c_{0}+c_{1} \alpha+c_{2} \alpha^{2}+c_{3} \alpha^{3}\right)<Q_{\max }
$$

where $Q_{\max }$ represents the acceptable maximum heating rate. $c_{0}, c_{1}$ and $c_{3}$ are constants. During the entire flight, two additional path constraints, dynamic pressure $P_{d}$ and normal acceleration $n_{L}$, are taken into account. These constraints are formulated as:

$$
\begin{aligned}
& P_{d}=\frac{1}{2} \rho V^{2}<P_{d \max } \\
& n_{L}=\frac{\sqrt{L^{2}+D^{2}}}{m g}<n_{L \max }
\end{aligned}
$$


145 load factor, respectively.

It should be noted that during the exo-atmospheric flight, the effects caused by heating rate, dynamic pressure and normal acceleration constraints are small and can be ignored (the dynamic pressure and normal acceleration are largely affected by the aerodynamic forces, whereas the heating rate constraint is largely affected by the density of the atmosphere). Therefore, these three constraints are removed from the mathematical model in the exo-atmospheric flight phases.

\subsection{Objective function}

In this mission scenario to ensure the aeroassisted vehicle has enough fuel to carry-out several skip hops, the objective is set to minimize the fuel consumption, i.e., maximize the final mass value, during the whole manoeuvre. More precisely, the objective function selected for the analysis is

$$
\min J=-m\left(t_{f}\right)
$$

\subsection{Optimal control problem formulation}

Based on the dynamic model, mission constraints and objective function stated in this section, an aeroassisted spacecraft orbital hopping optimal control problem can be constructed. The dynamic models given by Eq. (1) and Eq.(3) are abbreviated as $\dot{x}(t)=f(x(t), u(t))$, where $x=[r, \theta, \phi, V, \gamma, \psi, m]^{T} \in \Re^{7}$ and $u=[\alpha, \sigma]^{T} \in \Re^{2}$ denote the state and control variables, respectively. The general form of the orbital hopping optimal control formulation is then summarised

$$
\begin{array}{cl}
\text { Find } & x=x(t), u=u(t) \\
\text { minimize } & J=-m\left(t_{f}\right) \\
\text { subject to } & \forall t \in\left[t_{0}, t_{f}\right] \\
& \dot{x}(t)=f(x(t), u(t)) \\
& C(x(t), u(t)) \leq 0 \\
& \Phi\left(t_{0}, t_{i}, t_{f}, x_{0}, x_{i}, x_{f}\right)=0 \\
& x_{\min } \leq x \leq x_{\max } \\
& u_{\min } \leq u \leq u_{\max }
\end{array}
$$

where $C(\cdot)$ represents the flight path constrains given by Eq. (8) and Eq. (9). $\Phi$ is the boundary constraints (e.g. Eq.44) and Eq.(5)). 


\section{Pseudospectral method for solving optimal control problems}

In this paper, the optimal control problem given by Eq.111) is solved via the Gauss Pseudospectral Method (GPM) [17]. The motivation of using pseudospectral method relies on its ability in achieving high approximation accuracy which is usually an important factor to measure the effectiveness of the algorithm 4. Compared with other typical direct transcription algorithms, pseudospectral methods apply global polynomials and can achieve a higher accuracy with much less temporal nodes. A detailed analysis in terms of the approximation error order of pseudospectral methods can be found in [21. For completeness, a brief description of the GPM is stated in this section.

To apply the GPM, the time domain should be transformed from $\left[t_{0}, t_{f}\right]$ to $[-1,1]$ via $\tau=\frac{2 t}{t_{f}-t_{0}}-\frac{t_{f}+t_{0}}{t_{f}-t_{0}}$. Then the state and control are approximated using the Lagrange interpolation polynomials $L_{j}(\tau)$

$$
\begin{aligned}
& x(\tau) \approx \sum_{j=0}^{N_{k}} x\left(\tau_{j}\right) L_{j}(\tau) \\
& u(\tau) \approx \sum_{j=1}^{N_{k}} u\left(\tau_{j}\right) L_{j}(\tau)
\end{aligned}
$$

where $j \leq N_{k}, N_{k}$ is the number of temporal nodes. Take the derivative of Eq. 12 results in the following form:

$$
\frac{d x(\tau)}{d \tau} \approx \sum_{j=0}^{N_{k}} \frac{d L_{j}(\tau)}{d \tau} x\left(\tau_{j}\right)=\sum_{j=0}^{N_{k}} D_{k j} x\left(\tau_{j}\right)
$$

where $k=1, . ., N_{k}$ and $D_{k j}$ denotes the elements of the $N_{k} \times\left(N_{k}+1\right)$ differentiation matrix [21] and it can be computed by:

$$
D_{k j}=\frac{d L_{j}\left(\tau_{k}\right)}{d \tau}=\sum_{m=0}^{N_{k}} \frac{\prod_{l=0, l \neq j, m}\left(\tau_{k}-\tau_{l}\right)}{\prod_{l=0, l \neq j}\left(\tau_{j}-\tau_{l}\right)}
$$

Based on Eq. 121-14], the dynamic equations in Eq. (11] is transcribed into algebraic equations:

$$
\sum_{j=0}^{N_{k}} D_{k j} x\left(\tau_{j}\right)-\frac{t_{f}-t_{0}}{2} f\left(x_{k}, u_{k}\right)=0
$$

where $x_{k} \equiv x\left(\tau_{k}\right)$ and $u_{k} \equiv u\left(\tau_{k}\right)$. Specifically, take the exo-atmospheric flight dynamics as an example (given by Eq.(1)), the equations of motion can be 
approximated by:

$$
\left\{\begin{array}{l}
\dot{r} \approx \sum_{j=0}^{N_{k}} D_{k j} r_{k} \\
\dot{\theta} \approx \sum_{j=0}^{N_{k}} D_{k j} \theta_{k} \\
\dot{\phi} \approx \sum_{j=0}^{N_{k}} D_{k j} \phi_{k} \\
\dot{V} \approx \sum_{j=0}^{N_{k}} D_{k j} V_{k} \\
\dot{\gamma} \approx \sum_{j=0}^{N_{k}} D_{k j} \gamma_{k} \\
\dot{\psi} \approx \sum_{j=0}^{N_{k}} D_{k j} \psi_{k} \\
\dot{m} \approx \sum_{j=0}^{N_{k}} D_{k j} m_{k}
\end{array}\right.
$$

The transformed algebraic equations can then be obtained via Eq. 13)-(15).

That is, for any $k=1, \ldots, N_{k}$,

$$
\begin{aligned}
& \sum_{j=0}^{N_{k}} D_{k j} r_{k}-\frac{t_{f}-t_{0}}{2} V_{k} \sin \gamma_{k}=0 \\
& \sum_{j=0}^{N_{k}} D_{k j} \theta_{k}-\frac{t_{f}-t_{0}}{2} \frac{V_{k} \cos \gamma_{k} \sin \psi_{k}}{r_{k} \cos \phi_{k}}=0 \\
& \sum_{j=0}^{N_{k}} D_{k j} \phi_{k}-\frac{t_{f}-t_{0}}{2} \frac{V_{k} \cos \gamma_{k} \cos \psi_{k}}{r_{k}}=0 \\
& \sum_{j=0}^{N_{k}} D_{k j} V_{k}-\frac{t_{f}-t_{0}}{2}\left(\frac{T \cos \alpha_{k}}{m}-g \sin \gamma_{k}+\omega_{V_{k}}\right)=0 \\
& \sum_{j=0}^{N_{k}} D_{k j} \gamma_{k}-\frac{t_{f}-t_{0}}{2}\left(\frac{T \sin \alpha}{m_{k} V}+\left(\frac{V_{k}^{2}-g r_{k}}{r_{k} V_{k}}\right) \cos \gamma_{k}+\omega_{\gamma_{k}}\right)=0 \\
& \sum_{j=0}^{N_{k}} D_{k j} \psi_{k}-\frac{t_{f}-t_{0}}{2}\left(\frac{V_{k}}{r_{k}} \cos \gamma_{k} \sin \psi_{k} \tan \phi_{k}+\omega_{\psi_{k}}\right)=0 \\
& \sum_{j=0}^{N_{k}} D_{k j} m_{k}+\frac{t_{f}-t_{0}}{2} \frac{T}{I_{s p} g}=0
\end{aligned}
$$

Eq.17) will be entailed in the optimization model as equality constraints.

Similarly, the path constraints are parameterized at these temporal nodes. After the pseudospectral discretization, the aeroassisted vehicle orbital hopping 


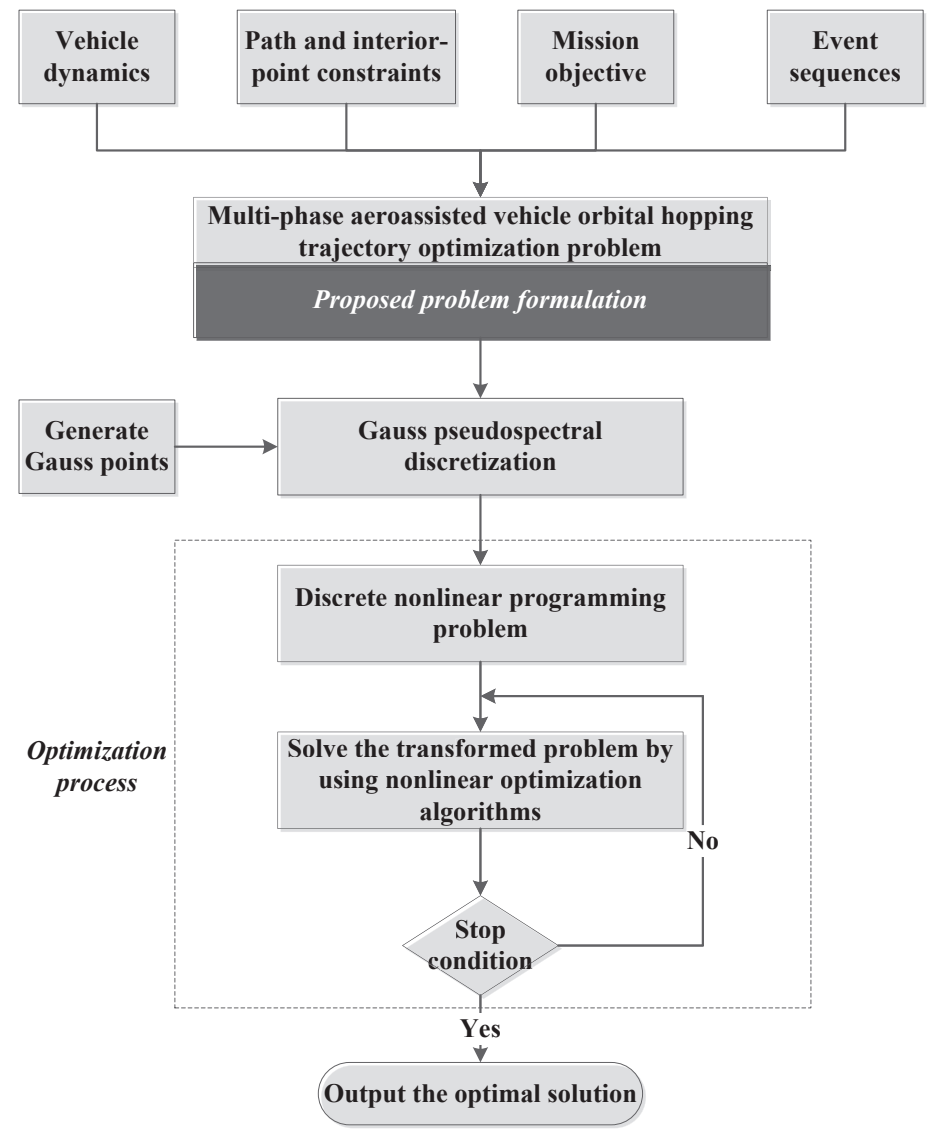

Figure 2: Overall structure of the algorithm

optimal control problem is transformed to a static Nonlinear Programming problem (NLP) [18, 26, 27]. The resulting NLP formulation can be solved by using optimization techniques [28]. There are many feasible and effective optimization algorithms that can be applied to solve the transcribed problem. For example, the evolutionary-based techniques [28], dynamic programming-based algorithms 200 [25], and the gradient-based optimization approaches [4]. A detailed comparative study regarding the performance of different optimization techniques can be found in 24, wherein a new two-nested gradient-based algorithm was also reported to calculate the optimal solution. This nonlinear optimization algorithm combines the advantages of interior point method (IP) and sequential quadratic programming (SQP). More precisely, this techniques contains two steps that 
solves a quadratic programming problem in the inner IP loop at a fixed index of the outer SQP loop.

A flowchart which illustrates the overall process to generate the optimal trajectories is plotted in Fig.2, whereas the general steps of the optimization process are summarised and tabulated in Table.1.

Table 1: Steps of the optimization process (gradient-based methods)

\begin{tabular}{|c|c|}
\hline Step No. & \\
\hline Step 1 & Initialize all the parameters for the optimization algorithm. \\
\hline Step 2 & $\begin{array}{l}\text { Check stopping conditions for the optimization loop; if not satisfy, go } \\
\text { to Step } 3 .\end{array}$ \\
\hline Step 3 & Construct the static NLP and solve it via Newton iteration. \\
\hline Step 4 & $\begin{array}{l}\text { Check the stopping condition for the inner optimization loop; if satisfy, } \\
\text { go to Step } 5 \text {. }\end{array}$ \\
\hline Step 5 & Calculate the step length and update the solution. \\
\hline Step 6 & Set the iteration number Iter $=$ Iter +1 and go back to Step 2 . \\
\hline
\end{tabular}

Remark 1. Currently, there are many effective direct transcription and optimization techniques that can be applied to solve the general trajectory optimization problems. In this paper, we only discuss and verify the formulation design of the aeroassisted spacecraft orbital hopping problem. A detailed comparison and analysis between different type of solution-finding techniques is beyond the scope of this paper. We refer to [24, 29] for such a comparison.

\section{Simulation results}

To investigate the feasibility and effectiveness of the proposed problem formulation (see Section.2), a number of simulation experiments were carried out. The aeroassisted spacecraft orbital hopping problem was solved for four mission scenarios. For each mission scenario, it contains $n=(1,2,3,4)$ atmospheric hops. The four target position information (target boundary conditions) is tabulated in Table.2. The initial state boundary conditions of the aeroassisted orbital transfer vehicle [30] are set as $x_{0}=\left[r_{0}, \theta_{0}, \phi_{0}, V_{0}, \gamma_{0}, \psi_{0}, m_{0}\right]=[120 \mathrm{~km}, 0 \mathrm{deg}, 0 \mathrm{deg}$, ${ }_{225} 7802.9 \mathrm{~m} / \mathrm{s},-1 \mathrm{deg}, 90 \mathrm{deg}, 818 \mathrm{~kg}$ ]. In the unpowered atmospheric phase, the decision variable $m$ is treated as a constant. 
Table 2: Target position information

\begin{tabular}{|c|c|c|c|c|}
\hline Parameter & $n_{1}$ & $n_{2}$ & $n_{3}$ & $n_{4}$ \\
$r_{i}(\mathrm{~km})$ & 60 & 60 & 60 & 60 \\
$\theta_{i}(\mathrm{deg})$ & 4.69 & 38.80 & 125.92 & 159.02 \\
$\phi_{i}(\mathrm{deg})$ & 12.60 & 59.70 & 65.65 & 44.28 \\
$\gamma_{i}(\mathrm{deg})$ & 0 & 0 & 0 & 0 \\
\hline
\end{tabular}

It is worth mentioning that in this study, a point mass model is used to represent the vehicle, which means only the translational motion is considered in the design of trajectory. This assumption may result in some limitations or negative effects. For example, when vibrations and other oscillatory effects are taken into account, the obtained control histories may have high-frequency fluctuations, which is undesired in the practical trajectory control system [31. According to the authors' previous investigation [29, this issue can be alleviated using time lags and the result shows that it is generally robust to apply the point mass model for generating the flight trajectory. However, if it is desired to not only design the trajectory but also achieve the attitude control, this assumption does not hold and a rigid body model should be used to calculate the control profiles.

In terms of the flight path constraints, the maximum allowable heating, dynamic pressure and load factor are set as: $Q_{\max }=200 \mathrm{Btu} / \mathrm{ft}^{2} \cdot \mathrm{s}\left(227 \mathrm{~W} / \mathrm{cm}^{2}\right)$; $P d_{\max }=13406.4583 P a ; n l_{\max }=2.5$, respectively. $T=2500 \mathrm{~N}, N_{k}$ is set to 40 for each phase. Other less important vehicle-dependent and mission-dependent parameters can be found in [22, 23. All the numerical simulations were executed under Windows 7 and Intel(R) i7-4790 CPU, 2.90GHZ, with 4.00 GB RAM.

\subsection{Multiple regional reconnaissance results}

The overall optimal solutions are provided for different orbital hopping scenarios. Fig.3 to Fig.6 illustrate the optimal state and path constraint time histories for $n=1,2,3,4$ scenarios.

Since maximizing the final mass value is chosen as the objective function, the optimization solver will minimize the time duration of using the engine model. As a result, it can be expected that the optimal solution tends to contain fewer exo-atmospheric phases (shown in Figs.3-6). It is worth noting that in Figs.3-5, the additional skip-entry is used to adjust the attitude of the aeroas- 

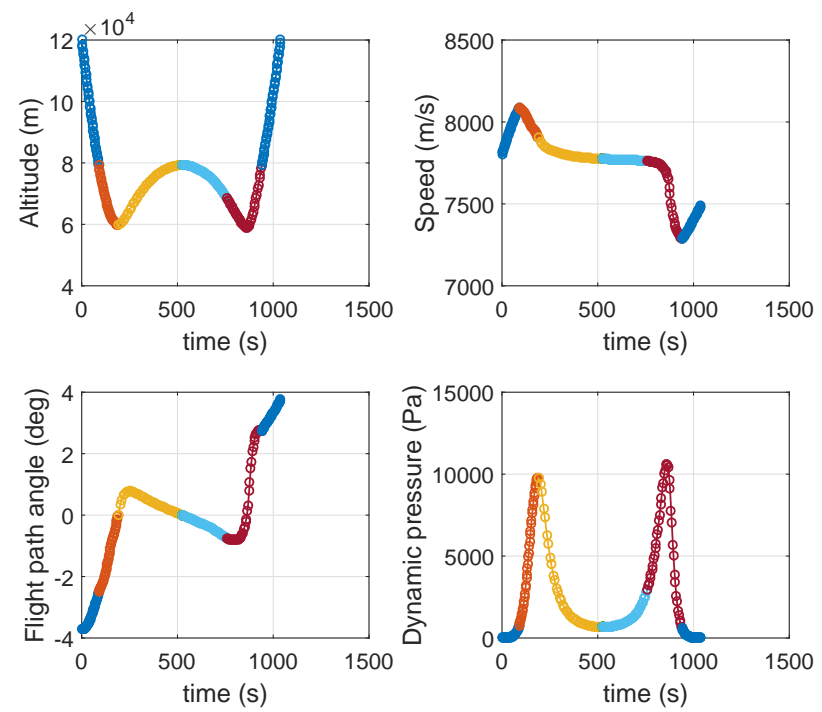

Figure 3: State and constraint time history: $\mathrm{n}=1 ; Q_{\max }=200 B t u / f t^{2} \cdot s\left(227 \mathrm{~W} / \mathrm{cm}^{2}\right)$; $P d_{\max }=13406.4583 P a ; n l_{\max }=2.5$

sisted vehicle so that it can have a higher value of flight path angle at the exit point. In this way, the vehicle can achieve the final boundary condition within short time in the final powered exo-atmospheric phase, thereby maximizing the final mass value indirectly.

The fuel consumption is shown by introducing a mass fraction indicator $m_{f} / m_{0}$, where $m_{f}$ stands for the mass value at $t_{f}$. Fig.7 illustrates the relationship between the final mass fraction and the number of $n$ (skip hops). As can be seen from Fig.7, the difference between the final mass fraction for $n=1$ and $n=2$ cases are small. However, more fuel is consumed for the $n=3$ and $n=4$ cases. This can be explained that for $n<3$ cases, to overfly the target positions and save more fuel, the vehicle mainly uses the aerodynamic forces (e.g. aerodynamic drag and lift) to maneuver. When $n$ becomes larger (e.g. $n=3,4$ ), it tends to be more difficult for the flight vehicle to satisfy the mission requirements due to the loss of kinetic energy (see Fig.6). This indicates the vehicle needs to use the powered exo-atmospheric flight phase to compensate the loss of speed. Therefore, it can be concluded that the fuel fraction becomes higher with the increasing of $n$.

Based on the results shown in Figs.3-7, it can be observed that the design 

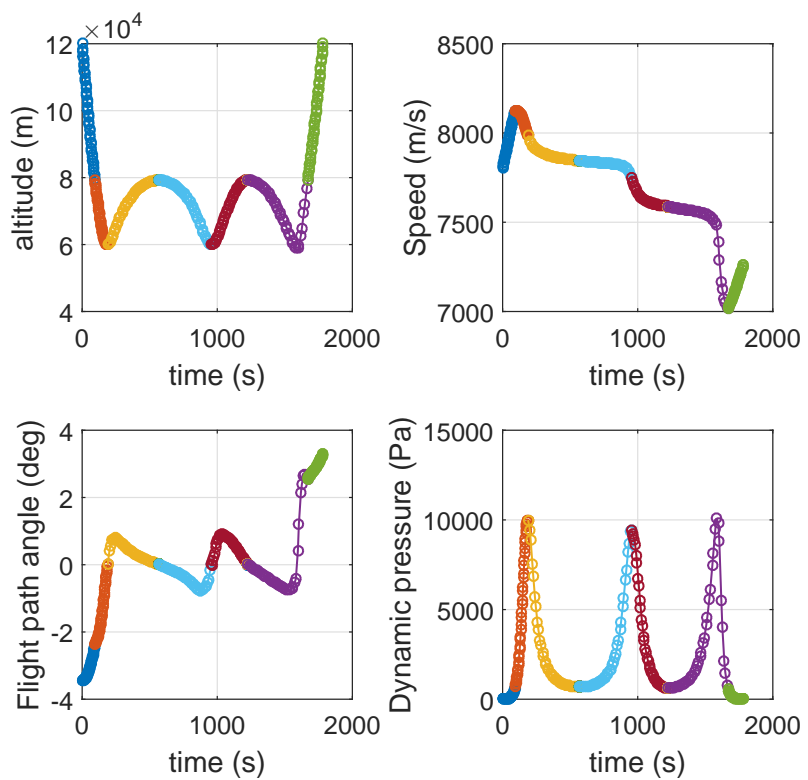

Figure 4: State and constraint time history: $\mathrm{n}=2 ; Q_{\max }=200 B t u / f t^{2} \cdot s\left(227 \mathrm{~W} / \mathrm{cm}^{2}\right)$; $P d_{\max }=13406.4583 P a ; n l_{\max }=2.5$

and formulation of the aeroassisted spacecraft orbital hopping problem stated in Section.2 can generate physically meaningful solutions and achieve the mission requirements successfully.

Remark 2. In order to use the engine model efficiently, the engine is off during the atmospheric flight. An advantage of this design is that the negative effects caused by aerodynamic forces in the acceleration phase can be eliminated. More precisely, in the $\dot{V}$ equation, only the term $\frac{T \cos \alpha}{m}$ is included in the acceleration phase (exo-atmospheric phases) instead of $\frac{T \cos \alpha-0.5 \rho V^{2} S C_{D}}{m}[22,23]$.

\subsection{Analysis of different skip hopping scenarios}

Solutions obtained for different orbital hopping mission scenarios are now investigated. Fig. 8 shows the altitude versus velocity histories during the atmospheric flight phase for $n=1,2,3,4$, while Fig.9 illustrates the corresponding time histories of the altitude and flight path angle.

285

Interestingly, it is shown in Fig. 8 that the velocity of the vehicle at the start of any intermediate atmospheric phase is larger than the velocity at the 

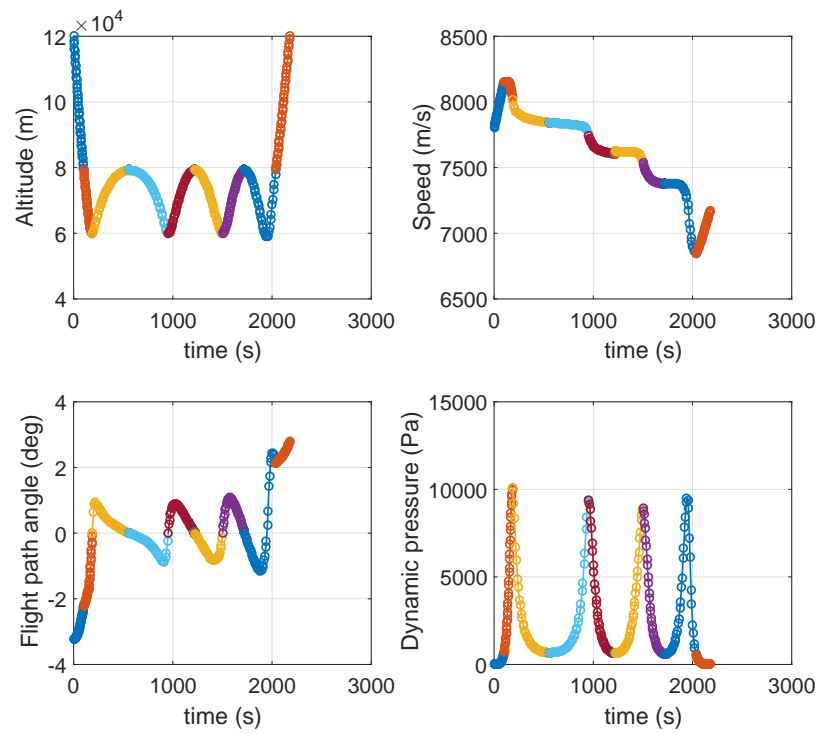

Figure 5: State and constraint time history: $\mathrm{n}=3 ; Q_{\max }=200 \mathrm{Btu} / \mathrm{ft} \mathrm{t}^{2} \cdot \mathrm{s}\left(227 \mathrm{~W} / \mathrm{cm}^{2}\right)$; $P d_{\max }=13406.4583 P a ; n l_{\max }=2.5$
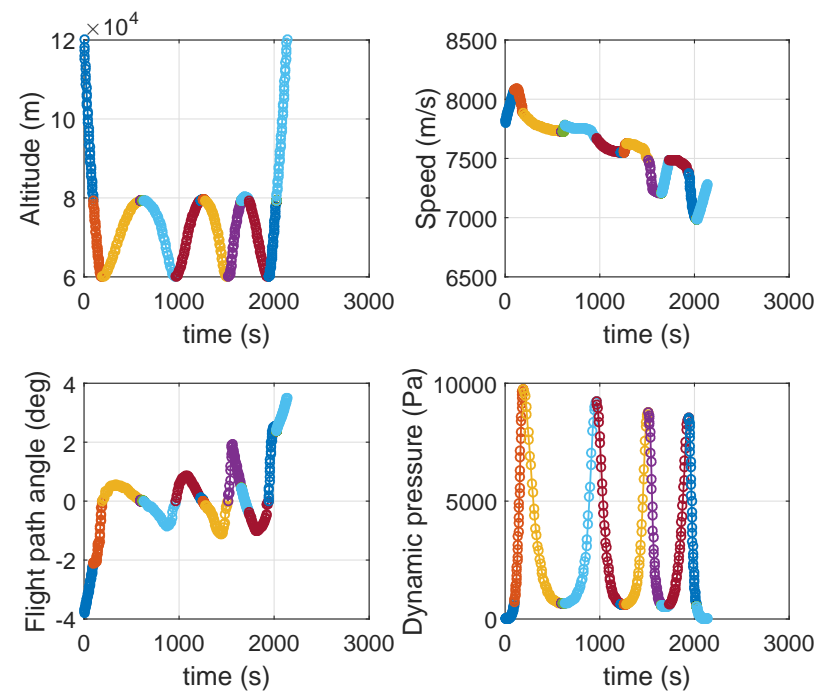

Figure 6: State and constraint time history: $\mathrm{n}=4 ; Q_{\max }=200 \mathrm{Btu} / \mathrm{ft} \mathrm{t}^{2} \cdot \mathrm{s}\left(227 \mathrm{~W} / \mathrm{cm}^{2}\right)$; $P d_{\max }=13406.4583 P a ; n l_{\max }=2.5$ 


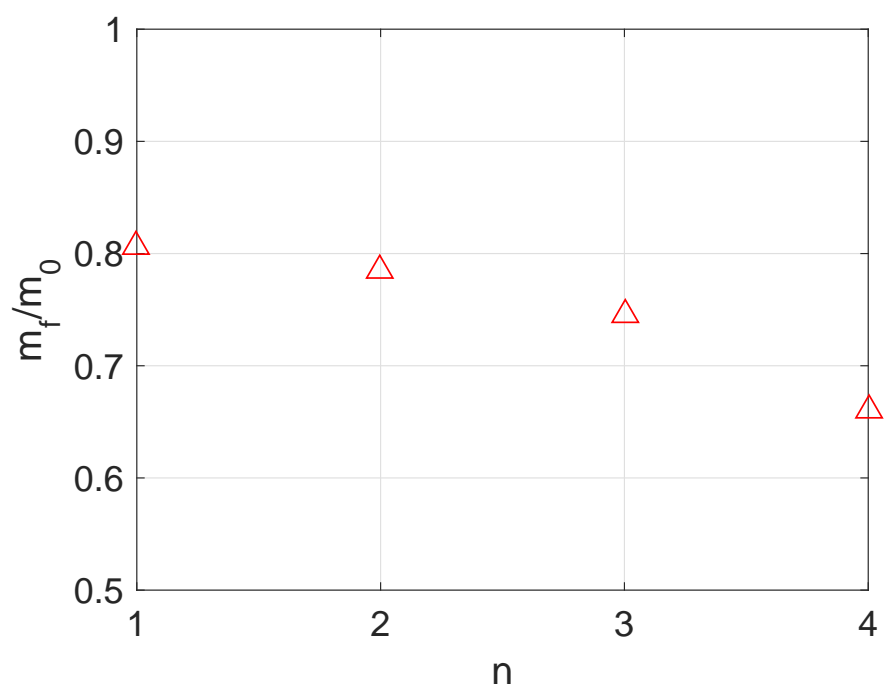

Figure 7: Final mass fraction versus $\mathrm{n}$
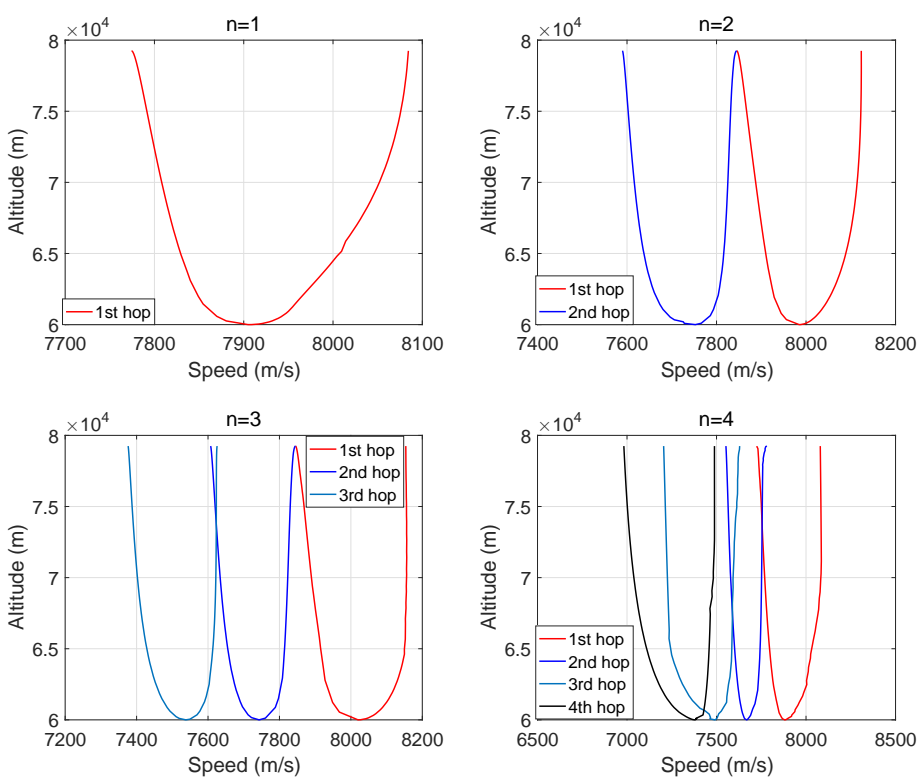

Figure 8: Altitude versus speed for $\mathrm{n}=1,2,3,4: Q_{\max }=200 \mathrm{Btu} / \mathrm{ft} t^{2} \cdot s\left(227 \mathrm{~W} / \mathrm{cm}^{2}\right)$ 

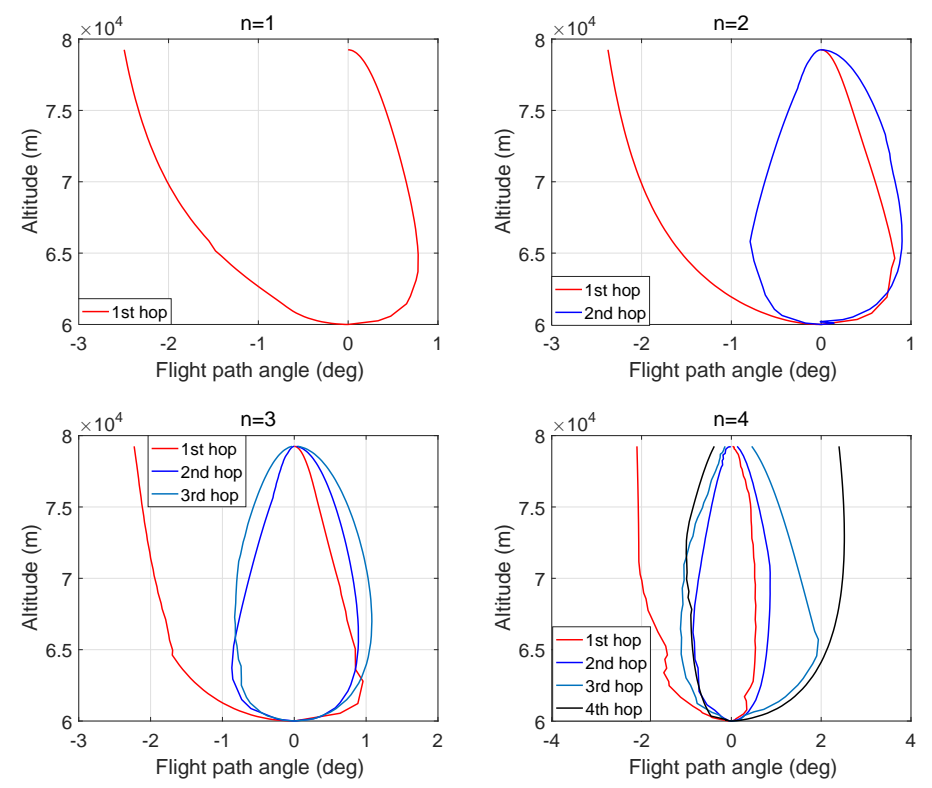

Figure 9: Altitude versus flight angle for $\mathrm{n}=1,2,3,4: Q_{\max }=200 \mathrm{Btu} / \mathrm{ft} t^{2} \cdot \mathrm{s}\left(227 \mathrm{~W} / \mathrm{cm}^{2}\right)$

terminus of the previous atmospheric pass for $n \geq 3$ cases. In other words, there are some mismatch points between the end and ensuring atmospheric hop phases for $n=3$ and $n=4$ cases. This result implies that an intermediate powered exo-atmospheric phase is added between the two orbital hopping phases in order to compensate the energy loss. This can also be found in Fig.9, where the flight path angle value at the end of an atmospheric flight does not go back to 0 .

For $n=1$ and $n=2$, the speed value at the start of an atmospheric entry keeps the same as the speed at the termination of the previous atmospheric entry, which means in these cases, the vehicle is able to only use the aerodynamic forces to complete the entire mission.

\subsection{Sensitivity with respect to path constraint}

It is well known that for spacecraft trajectory optimization, the optimal solution are largely affected by the flight path constrains. To analyze the sensitivity in terms of path constraints for the optimal solution, several comparative simulations were carried out. 
Firstly, attention is given to analyze the effects of the maximum allowable heating rate on the objective function (i.e. minimizing the fuel consumption). By setting $Q_{\max }=200 B t u / f t^{2} \cdot s\left(227 \mathrm{~W} / \mathrm{cm}^{2}\right), Q_{\max }=250 B t u / f t^{2}$.

$s\left(284 \mathrm{~W} / \mathrm{cm}^{2}\right), Q_{\max }=300 \mathrm{Btu} / \mathrm{ft} \mathrm{t}^{2} \cdot \mathrm{s}\left(340 \mathrm{~W} / \mathrm{cm}^{2}\right)$ and $Q_{\max }=\infty$ for $n=1,2,3$ and 4 cases, the results are calculated and plotted in Fig.10. From Fig.10,

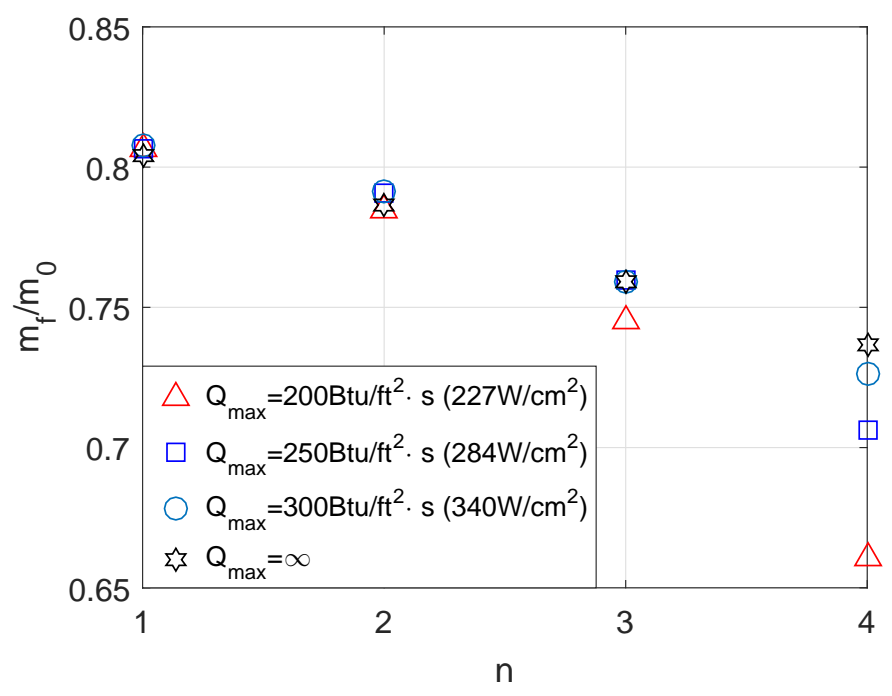

Figure 10: Final mass fraction for different $Q_{\max }: \mathrm{n}=4$

it can be observed that a strict heating constraint will result in a large final mass fraction. The mass fraction indicator tends to overlap for each scenario ( $n=1,2,3,4)$ when the heating constraint becomes easy to satisfy.

The results of a specific mission scenario $(n=4)$ are shown in Fig.11 and Fig.12 to illustrate the influences of the heating rate in terms of the vehicles's flight path and velocity.

It is worth mentioning that one effective way to avoid the heating constraint becoming active is to decrease the velocity significantly. When the heating con315 lose more kinetic energy during the atmospheric phases. Therefore, a powered exo-atmospheric flight phase can be found in the optimal trajectories to connect the two orbital hopping phases (see Fig.9 and Fig.12). On the other hand, if there is no constraint with respect to the heating rate, the aeroassisted vehicle can complete the entire mission without using additional powered exo- 

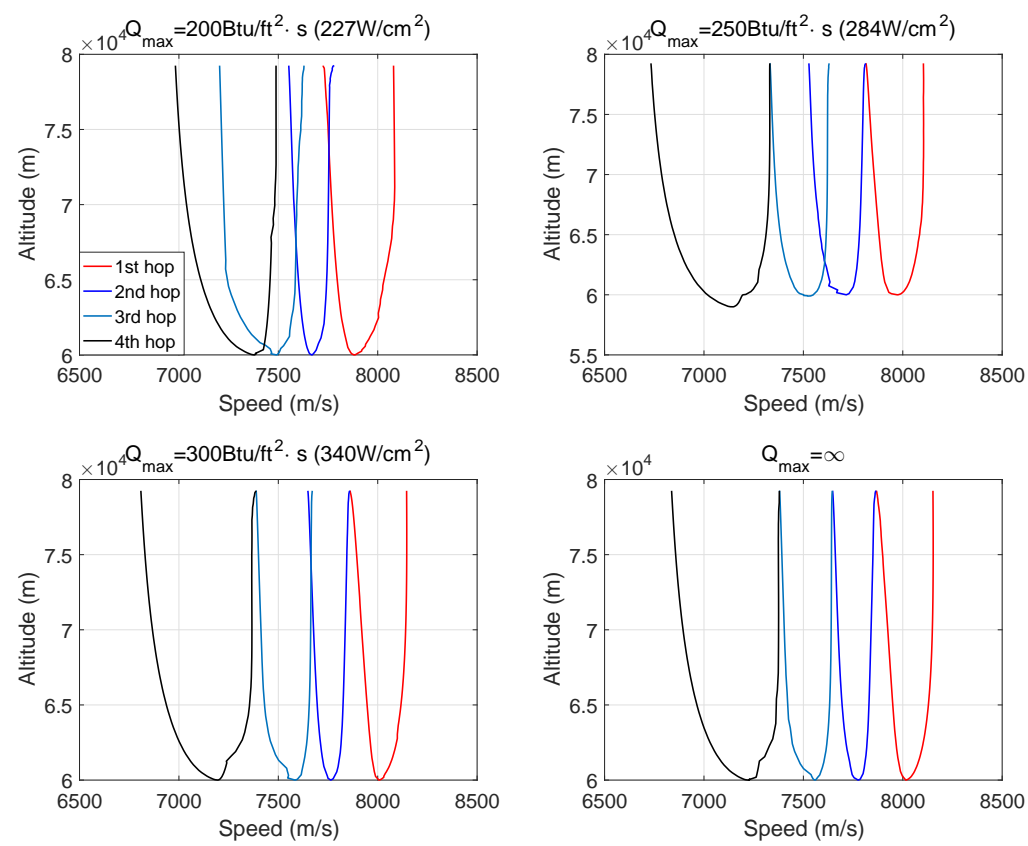

Figure 11: Altitude versus speed for different $Q_{\max }: \mathrm{n}=4$

atmospheric phases. This can also be reflected in Fig.11 and Fig.12, where there is no mismatch point in the vehicle's velocity and flight path angle profiles for the $Q_{\max }=\infty$ case.

In order to better show the effect of $Q_{\max }$ on the actual flight phases,

325 Table. 3 is constructed to show the thrust durations during each powered exoatmospheric segment alongside the total propulsive time $\Delta t_{\text {pro }}$ in all cases $(n=$ $1,2,3,4)$ for different $Q_{\max }$ values. Correspondingly, Table.4 provides the data in terms of the unpowered atmospheric flight durations $\Delta t_{a t m}$ for all cases. According to the data provided in Table. 3 and Table.4, it is obvious that the frequency of using the powered exo-atmospheric flight phase increases as $Q_{\max }$ decreases. Consequently, the time duration distribution shown in Table.4 is influenced significantly.

A comprehensive experiment and analysis in terms of the highly correlated or contradicting relationships between aerodynamic heat, vehicle's speed and 335 fuel consumption can be found in [22, 23]. 

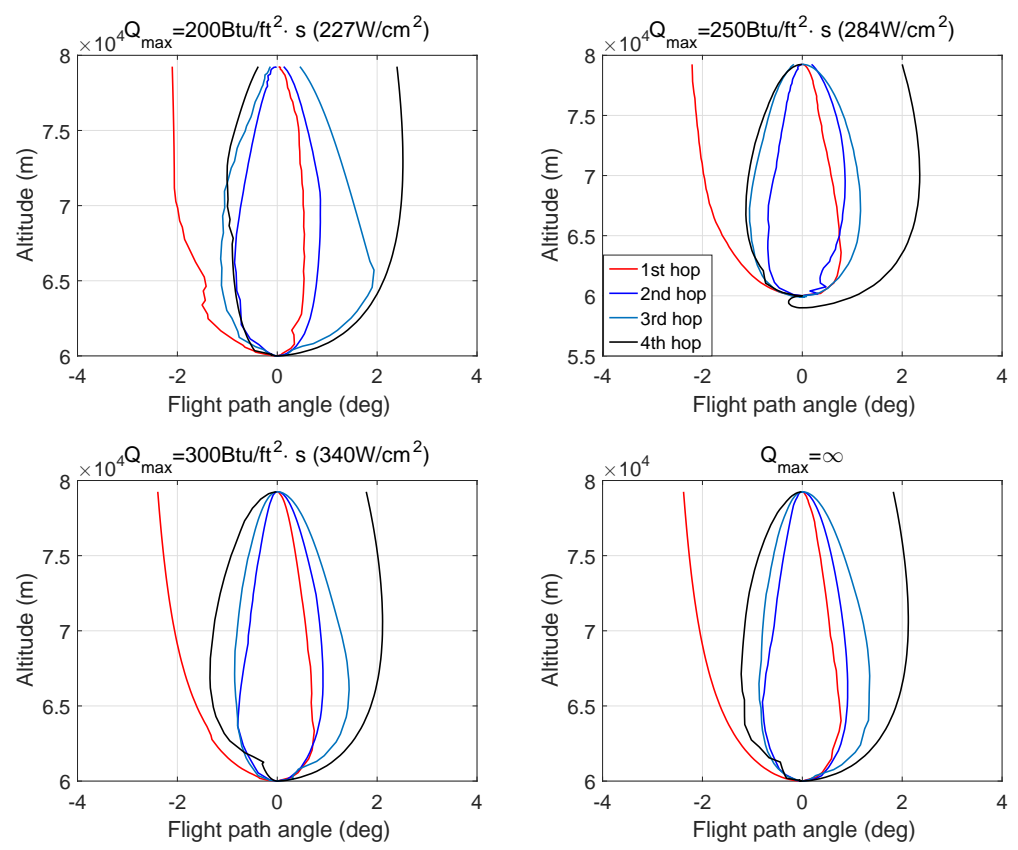

Figure 12: Altitude versus flight angle for different $Q_{\max }: \mathrm{n}=4$

\subsection{Finding solution for $n>4$ scenarios}

Extensive experiments were made to analyze the mission scenarios when $n$ is greater than 4. However, the current optimal control formulation stated in Section 2 failed to produce high quality solutions that can meet the mission requirements and optimize the performance index without violating all the constraints. This is because the size of the problem and number of optimization parameters tend to increase as $n$ increases. Moreover, the mission constraints, especially for the interior-point constraints used to keep the continuity between different mission phases, become more difficult to satisfy. Following a large amount of solution-finding iterations, the optimization solver still failed to catch the behaviour of the equations of motion and satisfy all the mission requirements. Therefore, it is suggested that the original problem should be divided into smallscale subproblems for $n>4$ cases. Then the terminal conditions obtained from the previous subproblem solution are applied as the initial conditions for the following subproblem, and the optimization program is restarted. For example, if it is desired to have an optimal trajectory for the $n=7$ case, this issue can 
Table 3: Thrust phase durations for all cases $\Delta t_{\text {pro }}$, seconds (s)

\begin{tabular}{|c|c|c|c|c|c|c|}
\hline & $\Delta t_{\text {pro }}^{(1)}(\mathrm{s})$ & $\Delta t_{\text {pro }}^{(2)}(\mathrm{s})$ & $\Delta t_{\text {pro }}^{(3)}(\mathrm{s})$ & $\Delta t_{\text {pro }}^{(4)}(\mathrm{s})$ & $\Delta t_{\text {pro }}^{(5)}(\mathrm{s})$ & $\Delta t_{\text {pro }}^{(6)}(\mathrm{s})$ \\
\hline$Q_{\max }$ & & & $n=1$ & & & \\
\hline 200 & 89.39 & 0 & 101.10 & - & - & - \\
\hline 250 & 89.16 & 0 & 97.89 & - & - & - \\
\hline 300 & 88.05 & 0 & 97.41 & - & - & - \\
\hline$\infty$ & 87.93 & 0 & 97.63 & - & - & - \\
\hline$Q_{\max }$ & & & $n=2$ & & & \\
\hline 200 & 95.03 & 0 & 0 & 112.90 & - & - \\
\hline 250 & 93.34 & 0 & 0 & 108.64 & - & - \\
\hline 300 & 94.31 & 0 & 0 & 107.06 & - & - \\
\hline$\infty$ & 95.99 & 0 & 0 & 110.29 & - & - \\
\hline$Q_{\max }$ & & & $n=3$ & & & \\
\hline 200 & 101.47 & 0 & 5.6 & 0 & 139.10 & - \\
\hline 250 & 97.83 & 0 & 0 & 0 & 135.06 & - \\
\hline 300 & 97.27 & 0 & 0 & 0 & 134.81 & - \\
\hline$\infty$ & 96.10 & 0 & 0 & 0 & 136.70 & - \\
\hline$Q_{\max }$ & & & $n=4$ & & & \\
\hline 200 & 97.67 & 17.64 & 23.83 & 77.12 & 0 & 112.35 \\
\hline 250 & 97.62 & 0 & 31.80 & 0 & 0 & 154.75 \\
\hline 300 & 97.87 & 0 & 6.86 & 0 & 0 & 159.80 \\
\hline$\infty$ & 99.01 & 0 & 0.03 & 0 & 0 & 155.59 \\
\hline
\end{tabular}

be solved effectively by constructing two subproblems $n=4$ and $n=3$.

\section{Conclusions}

In this work, a constrained minimum-fuel aeroassisted spacecraft orbital hopping mission has been constructed and studied. The entire mission was transcribed into a nonlinear multi-phase optimal control problem and solved applying a well-developed direct transcription algorithm. In order to guide the aeroassisted vehicle overflying different target positions, a series of event sequences was constructed and embedded in the optimal control formulation. A couple of interior-point constraints were also introduced so as to enhance the continuity of the trajectory between different flight phases. Comparative simulations indicated that the proposed formulation design can produce feasible 
Table 4: Atmospheric flight durations for all cases $\Delta t_{a t m}$, seconds (s)

\begin{tabular}{|c|c|c|c|c|c|c|}
\hline & $\Delta t_{a t m}^{(1)}(\mathrm{s})$ & $\Delta t_{a t m}^{(2)}(\mathrm{s})$ & $\Delta t_{a t m}^{(3)}(\mathrm{s})$ & $\Delta t_{\text {atm }}^{(4)}(\mathrm{s})$ & $\Delta t_{a t m}^{(5)}(\mathrm{s})$ & $\Delta t_{a t m}(\mathrm{~s})$ \\
\hline$Q_{\max }$ & & & $n=1$ & & & \\
\hline 200 & 438.21 & 413.12 & - & - & - & 851.33 \\
\hline 250 & 434.44 & 413.19 & - & - & - & 847.63 \\
\hline 300 & 428.42 & 481.19 & - & - & - & 909.61 \\
\hline$\infty$ & 436.52 & 412.54 & - & - & - & 849.06 \\
\hline$Q_{\max }$ & & & $n=2$ & & & \\
\hline 200 & 472.79 & 658.43 & 0 & 112.90 & - & 1573.74 \\
\hline 250 & 476.94 & 661.88 & 0 & 108.64 & - & 1494.09 \\
\hline 300 & 481.66 & 686.12 & 0 & 107.06 & - & 1558.46 \\
\hline$\infty$ & 487.10 & 657.78 & 0 & 110.29 & - & 1750.32 \\
\hline$Q_{\max }$ & & & $n=3$ & & & \\
\hline 200 & 454.80 & 659.31 & 491.97 & 320.14 & 139.10 & 1926.22 \\
\hline 250 & 475.57 & 647.40 & 516.70 & 317.71 & 135.06 & 1957.38 \\
\hline 300 & 479.36 & 646.49 & 501.98 & 317.87 & 134.81 & 1945.70 \\
\hline$\infty$ & 480.94 & 647.10 & 501.38 & 317.65 & 136.70 & 1947.07 \\
\hline$Q_{\max }$ & & & $n=4$ & & & \\
\hline 200 & 494.79 & 593.67 & 378.11 & 290.03 & 0 & 1756.66 \\
\hline 250 & 468.31 & 633.33 & 446.91 & 355.99 & 0 & 1904.54 \\
\hline 300 & 472.12 & 644.37 & 465.94 & 328.20 & 0 & 1910.63 \\
\hline$\infty$ & 487.32 & 630.70 & 484.66 & 319.18 & 0 & 1912.86 \\
\hline
\end{tabular}

flight trajectories that can achieve different mission requirements and minimize the overall fuel consumption. Also, other key features of the obtained optimal solution, including the relationship between the mass fraction and number of hops, and the sensitivity with respect to path constraints, have also been analyzed.

There exist several topics left to be further investigated. For instance, since the point mass model used in this paper can be treated as an approximation of the rigid body model, the accuracy should be analyzed. Moreover, in practical trajectory control system, the vehicle dynamics may contain some uncertain variables and disturbances. In this case, this mission needs to be formulated as a stochastic trajectory optimization problem and stochastic optimization techniques should be designed to solve it. These will be the subjects of our follow-up research. 


\section{Acknowledgments}

The authors would like to thank the editors and all the reviewers for their valuable comments and suggestions.

\section{References}

[7] B. Tian, Q. Zong, J. Wang, F. Wang, Quasi-continuous high-order slid-

[8] D. G. Hull, J. M. Giltner, J. L. Speyer, J. Mapar, Minimum energy-loss guidance for aeroassisted orbital plane change, Journal of Guidance, Con-

[1] A. V. Rao, S. Tang, W. P. Hallman, Numerical optimization study of multiple-pass aeroassisted orbital transfer, Optimal Control Applications and Methods 23 (4) (2002) 215-238. doi:10.1002/oca.711.

[2] T. R. Jorris, R. G. Cobb, Multiple method 2-D trajectory optimization satisfying waypoints and no-fly zone constraints, Journal of Guidance, Control, and Dynamics 31 (3) (2008) 543-553. doi:10.2514/1.32354.

[3] T. R. Jorris, R. G. Cobb, Three-dimensional trajectory optimization satisfying waypoint and no-fly zone constraints, Journal of Guidance, Control, and Dynamics 32 (2) (2009) 551-572. doi:10.2514/1.37030.

[4] C. L. Darby, W. W. Hager, A. V. Rao, Direct trajectory optimization using a variable low-order adaptive pseudospectral method, Journal of Spacecraft and Rockets 48 (3) (2011) 433-445. doi:10.2514/1.52136

[5] C. Chawla, P. Sarmah, R. Padhi, Suboptimal reentry guidance of a reusable launch vehicle using pitch plane maneuver, Aerospace Science and Technology 14 (6) (2010) 377-386. doi:http://dx.doi.org/10.1016/j.ast. 2010.04 .001

[6] J. Dai, Y. Xia, Mars atmospheric entry guidance for reference trajectory tracking, Aerospace Science and Technology 45 (2015) 335-345. doi:http: //dx.doi.org/10.1016/j.ast.2015.06.006

ing mode controller design for reusable launch vehicles in reentry phase, Aerospace Science and Technology 28 (1) (2013) 198-207. doi:http: //dx.doi.org/10.1016/j.ast.2012.10.015 trol, and Dynamics 8 (4) (1985) 487-493. doi:10.2514/3.20009 
[9] D. S. Naidu, J. L. Hibey, C. D. Charalambous, Neighboring optimal guidance for aeroassisted orbital transfer, IEEE Transactions on Aerospace and Electronic Systems 29 (3) (1993) 656-665. doi:10.1109/7.220918.

[10] C. Gogu, T. Matsumura, R. T. Haftka, A. V. Rao, Aeroassisted orbital transfer trajectory optimization considering thermal protection system mass, Journal of Guidance, Control, and Dynamics 32 (3) (2009) 927-938. doi:10.2514/1.37684.

[11] C. L. Darby, A. V. Rao, Minimum-fuel low-earth orbit aeroassisted orbital transfer of small spacecraft, Journal of Spacecraft and Rockets 48 (4) (2011) 618-628. doi:10.2514/1.A32011.

[12] B. Senses, A. V. Rao, Optimal finite-thrust small spacecraft aeroassisted orbital transfer, Journal of Guidance, Control, and Dynamics 36 (6) (2013) 1802-1810. doi:10.2514/1.58977.

[13] H. Wang, P. Shi, H. Li, Q. Zhou, Adaptive neural tracking control for a class of nonlinear systems with dynamic uncertainties, IEEE Transactions on Cybernetics 47 (10) (2017) 3075-3087. doi:10.1109/TCYB.2016.2607166

[14] J. Xu, P. Shi, C. C. Lim, C. Cai, Y. Zou, Integrated structural parameter and robust controller design for attitude tracking maneuvers, IEEE/ASME Transactions on Mechatronics 21 (5) (2016) 2490-2498. doi:10.1109/ TMECH . 2016.2570820

[15] X. Zhao, P. Shi, X. Zheng, J. Zhang, Intelligent tracking control for a class of uncertain high-order nonlinear systems, IEEE Transactions on Neural Networks and Learning Systems 27 (9) (2016) 1976-1982. doi:10.1109/ TNNLS.2015.2460236

[16] Q. Zhou, C. Wu, P. Shi, Observer-based adaptive fuzzy tracking control of nonlinear systems with time delay and input saturation, Fuzzy Sets and

(1) Systems 316 (Supplement C) (2017) 49-68. doi:https://doi.org/10. 1016/j.fss.2016.11.002.

[17] D. A. Benson, G. T. Huntington, T. P. Thorvaldsen, A. V. Rao, Direct 435 trajectory optimization and costate estimation via an orthogonal collocation method, Journal of Guidance, Control, and Dynamics 29 (6) (2006) 1435-1440. doi:10.2514/1.20478. 
[18] D. Garg, M. Patterson, W. W. Hager, A. V. Rao, D. A. Benson, G. T. Huntington, A unified framework for the numerical solution of optimal control problems using pseudospectral methods, Automatica 46 (11) (2010) 1843-1851. doi:http://dx.doi.org/10.1016/j.automatica.2010.06. 048.

[19] F. Fahroo, I. M. Ross, Direct trajectory optimization by a Chebyshev pseudospectral method, Journal of Guidance, Control, and Dynamics 25 (1) (2002) 160-166. doi:10.2514/2.4862.

[20] I. M. Ross, F. Fahroo, Pseudospectral knotting methods for solving nonsmooth optimal control problems, Journal of Guidance, Control, and Dynamics 27 (3) (2004) 397-405. doi:10.2514/1.3426.

[21] W. W. Hager, H. Hou, A. V. Rao, Convergence rate for a Gauss collocation method applied to unconstrained optimal control, Journal of Optimization Theory and Applications (2016) 1-24doi:10.1007/s10957-016-0929-7.

[22] R. Chai, A. Savvaris, A. Tsourdos, Fuzzy physical programming for space manoeuvre vehicles trajectory optimization based on hp-adaptive pseu1. dospectral method, Acta Astronautica 123 (2016) 62-70. doi:http: //dx.doi.org/10.1016/j.actaastro.2016.02.020.

[23] R. Chai, A. Savvaris, A. Tsourdos, S. Chai, Multi-objective trajectory optimization of space manoeuvre vehicle using adaptive differential evolution and modified game theory, Acta Astronautica 136 (2017) 273-280. doi:http://dx.doi.org/10.1016/j.actaastro.2017.02.023.

[24] R. Chai, A. Savvaris, A. Tsourdos, S. Chai, Y. Xia, Improved gradient-based algorithm for solving aeroassisted vehicle trajectory optimization problems,

口 Journal of Guidance, Control, and Dynamics (2017) 1-9 doi:10.2514/1. G002183.

[25] J. T. Betts, Practical Methods for Optimal Control and Estimation Using Nonlinear Programming, Cambridge University Press, 2009.

[26] P. Williams, Jacobi pseudospectral method for solving optimal control problems, Journal of Guidance, Control, and Dynamics 27 (2) (2004) 293297. doi:10.2514/1.4063. 
[27] P. Shi, Limit Hamilton-Jacobi-Isaacs equations for singularly perturbed zero-sum dynamic (discrete time) games, SIAM Journal on Control and Optimization 41 (3) (2002) 826-850. doi:10.1137/s036301290037908x.

[28] B. A. Conway, A survey of methods available for the numerical optimization of continuous dynamic systems, Journal of Optimization Theory and Applications 152 (2) (2012) 271-306. doi:10.1007/s10957-011-9918-z.

475 [29] R. Chai, A. Savvaris, A. Tsourdos, Violation learning differential evolutionbased hp-adaptive pseudospectral method for trajectory optimization of space maneuver vehicle, IEEE Transactions on Aerospace and Electronic Systems 53 (4) (2017) 2031-2044. doi:10.1109/TAES.2017.2680698

[30] N. Berend, S. Bertrand, C. Jolly, Optimization method for mission analysis 480 of aeroassisted orbital transfer vehicles, Aerospace Science and Technology 11 (5) (2007) 432-441. doi:http://dx.doi.org/10.1016/j.ast.2007. 01.007

[31] F. Imado, Y. Heike, T. Kinoshita, Research on a new aircraft point-mass model, Journal of Aircraft 48 (4) (2011) 1121-1130. doi:10.2514/1. 485 C000200. 
2018-02-03

\section{Optimal fuel consumption finite-thrust orbital hopping of aeroassisted spacecraft}

Chai, Runqi

Elsevier

Chai R, Savvaris A, Tsourdos A, Chai S, Xia Y, Optimal fuel consumption finite-thrust orbital hopping of aeroassisted spacecraft, Aerospace Science and Technology, Volume 75, April 2018, pp. 172-182

http://dx.doi.org/10.1016/j.ast.2017.12.026

Downloaded from Cranfield Library Services E-Repository 